\title{
COLOUR VISION
}

A SYMPOSIUM on the "Visual Problems of Colour" was held at the National Physical Coratory during September 23-25.

The opening session of the Symposium was devoted. to a commemoration of the pioneer American worker on vision, Selig Hecht, who died in 1947 just after the Cambridge Conference on colour vision in which he had taken a leading part. Notable advances since then in our knowledge of the visual pigments were reviewed by Prof. G. Wald (Harvard) - a former student and collaborator of Hecht's-in his "Selig Hecht Lecture" entitled "Retinal Chemistry and the Physiology of Vision". Wald's conception of the visual pigments as a class of conjugated proteins has in recent years developed in two major directions, and, thanks to the work of W. A. H. Rushton (Cambridge), a frustration which has bothered workers on human colour vision for a long time has now in great part been removed. To deal first with the latter development, it has been proved by a completely objective method-an elaboration of the method of fundus examination with an ophthalmoscop3-that photosensitive and bleachable pigments are in fact present in the foveal ratina of the eye of living man. In his paper Rushton described first determinations of the absorption difference spactra and the action spectra of two such pigments, and the progress made towards identifying them with the 'pigments' inferred from psychophysical studies on normal and colourdefective subjects. The spoctral curves of Rushton's two pigments have maxima at about 540 and $590 \mathrm{~m} \mu$ respectively. The still more difficult problem of finding by the direct, physical method a possible pigment with its spectral maximum in the bluewhich trichromatic ideas would suggest-remains outstanding. Only the 540 pigment was observable in the foveas of colour-defectives (dichromats) of the protanopic type, and the shape of the spectral curve of this pigment agreed well with the relative luminous efficiency curve of such subjects measured by the psychophysical method. In normals and in dichromats of the deuteranopic type, the same 540 pigment is present and, in addition, the 590 pigment, but in the deuteranope as compared with the normal the proportion of 590 to 540 pigment is greater, by a factor of about 2. This is striking direct evidence in favour of Fick's hypothesis that in deuteranopes both the 'red' and 'green' fundamental 'processes' (independent spectral sensitivities) of the normal eye are present-if in different relative amounts-and that the colour defect springs from their association together or 'fusion' so that they function as a single process. On the other hand, the explanation of protanopia as the simple lack of one fundamental process is confirmed by Rushton's results. The first steps towards exploiting the method to give information on the quantities of the pigments, their photosensitivities and the kinetics of their bleaching and regeneration have already been taken, and promise much. It should be added that the work on the fovea was preceded by similar observations on the extrafoveal retina, showing the presence there of the pigment of scotopic rod vision with properties conforming closely with those already known by other methods.

The increasing number of visual pigments for which there is evidence from studies of retinal extracts or intact retinæ was emphasized by R. A. Morton and G. A. J. Pitt (Liverpool). They note that some ten pigments, all based on retinene $e_{1}$ as the chromophone group but occurring in different animals, have wavelengths of maximal absorption, $\lambda_{\max }$., distributed over the range 478-524 $\mathrm{m} \mu$, and conclude that the classification of the protein constituents of the pigments, the opsins, into scotopsins and photopsins, with the members in each group having very nearly the same properties, may need revision. In his lecture Wald put the position in the following way. Of the several known storeo-isomeric forms of retinene ${ }_{1}$, only one, the 11-cis or neo-b isomer, combines with the opsins to give the visual pigments actually occurring. A second isomer, the 9-cis or iso-a form, will combine with the opsins in vitro to give the so-called isopigments, but these appear not to be used in actual eyes. Thus it must be differences in the opsins which account for variations in the $\lambda_{\max }$ of the naturally occurring retinene, pigments. Denton and Warren's discovery that cortain deep-sea fish have visual pigments with a $\lambda_{\max }$. at about $480 \mathrm{~m} \mu$, coupled with very recent observations on other species of fish by Wald (see Nature, Nov. 9, 1957, p. 969), make it appear that the properties of fish opsins, defined say by the $\lambda_{\max }$ of the corresponding pigment, show a gradation with the depth at which the fish are normally found, from $\lambda_{\max }=500 \mathrm{~m} \mu$ approx. for surface fish to $\lambda_{\max }=480 \mathrm{~m} \mu$ approx. for deep-sea (200 fathom) fish. The opsins here concerned are classed as scotopsins, but the photopsins may well show a similar wide spread of proporties and (Morton and Pitt) this may have implications for the pigments of colour vision. In human vision, the much greater rate of dark-adaptation of the photopic compared with the scotopie mechanism is paralleled by the more rapid regeneration, after bleaching, of the cone pigments (Rushton's observa. tions), and this difference has in turn a correspondence with the different rates of synthesis in vitro of the photopic and scotopic pigments of the chicken (iodopsin and rhodopsin) from retinene $\theta_{1}$ and photopsin and scotopsin respectively. Wald emphasized, however, that rate of synthesis does not necessarily distinguish photopsins and scotopsins. For example, alligator and frog visual pigments are rhodopsins, both having a $\lambda_{\max }$. at nearly $500 \mathrm{~m} \mu$, but their synthesis-rates differ widely. For the former, synthesis occurs with the high speed expected from photopic pigments, and the dark-adaptation of the alligator, tested physiologically, is correspondingly rapid.

The second major devslopment of Wald's conception of the visual pigme..t is the stereo-isomerism of the chromaphore and its implications. In her discussion of the mode of attachment of the chroma. phore to the opsin, Ruth Hubbard (Harvard) took as starting point Morton and Pitt's suggestion that a specific amino group of the opsin condenses with the aldehyde group of retinene ( 1 or 2 ) to give a Schiff base, which then, by addition of a hydrogen ion, is converted to the conjugate acid. The opsin surface near the amino group is assumed to be so shaped that only the two retinene isomers-neo- $b$ and iso-a-which in fact combine with opsin can conform geometrically to this surface and be linked with it by secondary valency bonds along the retinene 
side-chain so as to stabilize the conjugate acid in a non-acid environment and prevent its ultimate hydrolysis into opsin and retinene. At the same time, the secondary bonds reduce the lowest excitation energy of the conjugate acid to a value corresponding to the observed $\lambda_{\max }$. of the pigment. Hubbard assumes that the first result of light absorption is the isomerization of the chromaphore to the all-trans form which no longer 'fits' the protein surface. With the rupture of the secondary bonds, stabilization is lost, hydrolysis ensues and the final result is separation of opsin and retinene. For the exceptional case of squid rhodopsin $\left(\lambda_{\max }=493 \mathrm{~m} \mu\right)$ which in neutral or slightly acid solution is not bleached by light but is changed to an allied pigment, acid metarhodopsin, with $\lambda_{\max }=500 \mathrm{~m} \mu$, Hubbard's explanation is that light absorption results as before in isomerization of the chromaphore to the all-trans form, but that the surface of this particular opsin conforms sufficiently closely to the shapes of either neo-b or all-trans isomers for both to be retained in combination with the opsin. Some difficulties were raised on the assumed effect of the secondary linkages on the excitation energy, but the further working out of this theory will be of great interest. The situation of the products of bleaching of visual pigments also arose in the paper by E. J. Denton (Marine Biology Station, Plymouth), who had studied the absorption of polarized light of different wave-lengths incident along or across the end-organs of intact animal retinæ, before and after bleaching. $\mathrm{He}$ found, among other things, a persistent (but not unchanging) orientation of the bleached 'chromaphore' groups lasting for about one hour. In the discussion, the suggestion that this implied a continuing attachment of the bleached chromaphore group to the opsin was not supported.

The bleaching kinetics of the visual pigments of four species of fish were found by H. J. A. Dartnall (Medical Research Council) to follow a similar course to those of frog rhodopsin, and, using the absolute spectral photosensitivity curve for the latter pigment obtained by Schneider, Goodeve and Lythgoe in 1939, the corresponding curves for the four fish species were derived. One conclusion from these results was that the quantum efficiency of bleaching is independent of wave-length in the range studied (440-580 $\mathrm{m} \mu)$, just as for frog rhodopsin.

In two review papers, G. S. Brindley (Cambridge) and K. O. Donner (Helsinki) considered the bearing on colour vision of the latest work on the electroretinogram and the spiko discharges from retinal elements. New work on frog retinæ by a microelectrode method indicated that the slow (tenths of second) variations of potential difference across the retina recorded in the normal electroretinogram are generated in a single layer of the retina from "structures which penetrate the external limiting membrane, almost certainly the rods and cones". For the human electroretinogram, a distinction between slower responses attributable to the scotopic (rod) mechanism and faster ones attributable to the photopic (cone) mechanism is well established, but the efforts made to distinguish different photopic mechanisms of colour vision-mainly by studies of electroretinogram spectral sensitivity curves-have not led to unambiguous results and are not very promising. Since the electroretinogram is, on the whole, a simpie summation of responses from all parts of the large rotinal area illuminated, the study of colour discriminetion of a small area such as the fovea, in isolation, is likely to be defeated by the smallness of the signal obtained. By contrast, the thrusholds for spike discharges of individual retinal units obtained by the microelectrode method have led to Granit's familiar dominator and modulator spectral sensitivity curves, which may well contain much of the colour-vision story. Donner showed that once we leave the scotopic dominator curve which electro-physiological, photochemical and psychophysical methods all show to bo a fundamental non-composite spectral sensitivity, we have to question whether any recorded curve is not a resultant of several fundamental sensitivities arising perhaps from mixtures of different visual pigments in the same end-organs or from neural elaboration of the responses from end-organs containing different pigments. For bird retinæe, distortion of the curves by oil-drop colour filters in the cones is a further com. plication. Various methods must be applied to analyse recorded spactral sensitivity curves, and among recent procedures Donner described the twocolour threshold technique, and the use, as an indicator property, of the different directional sensitivities of rods and cones. Donner concludes that the broad, photopic dominator curve is produced exclusively by cons end-organs, but that it is probably a composite of more than one fundamental spactral sensitivity. The narrow modulator curves he attributes essentially to the effects of neural interaction which modify the responses of receptors with broader spectral sensitivity curves, comparable with the scotopic dominator. But he also suggests that the appəarance of a modulator curve in a particular spectral region probably means that there is in action a receptor with a broad sensitivity curve peaking at that place.

The psychophysical papers showed a recrudescence of interest in the laws and representative data of trichromatic colour-metching and heterochromatic brightness-matching. Extensive measurements of the colour-mixture functions for a $10^{\circ}$ field (Stiles and Burch, National Physical Laboratory, and N. I. Speranskaya, Moscow) have provided a possible basis for a new standard colorimetric eye appropriate to large-field colour-matching, and preliminary 'field trials' of the new data were discussed by $D$. B. Judd (National Bureau of Standards, Washington). Some small deviations from the additivity law for complete colour-matching were observed in the basic measurements. If appreciable deviations do occur, any selected set of colour-mixture curves must be a compromise, and for practical applications this should be one that works best for commonly occurring stimuli of continuous energy distributions, rather than for the mixtures of two or three monochromatic components normally used in measuring colourmixture functions. G. W. Wyszecki (National Rэsearch Council, Canada) described a preliminary attack on the formidable problem of a direct determ. ination of 'best compromise' functions from colourmatches on 115 accurately specified filter colours, and the solution by an electronic computer of an elaborate set of simultaneous equations in 32 throe-component unknowns. In a contribution on the breakdown of the additivity law in direct-comparison heterochromatic brightness-matching, which is quite distinct from the additivity law in complete colourmatching, S. G. Yurov (Moscow) emphasized that it is the approach to additivity rather than the defect from additivity which is difficult to explain. However, in heterochromatic brightness-matching by the flicker method, additivity is certainly nearly true for a small 
matching field. H. G. Sperling (U.S. Naval Medical Research Laboratory, New London) examined the assumption of standard colour theory that the relative luminous efficiency function $V_{\lambda}$ is a linear combination of the colour-mixture functions, measured for the same subjects. Using the flicker method for determining $V_{\lambda}$ he found the assumption substantially true for wave-lengths greater than about $520 \mathrm{~m} \mu$. but below that wave-length discrepancies of the order of 15 per cent appeared.

N. T. Fedorov (Leningrad) reported rather similar discrepancies. While the practical repercussions of these blemishes on the ideal linear and additive system of colorimetry may be slight, their causes are of considerable interest in visual theory. For a large matching field, some of the observed small breakdown in additivity (high intensities) is attributable to the intrusion of rod vision. Such effects may be expected to increase if the matching field is centred not on the fovea but on an extra-foveal point. M. M. Bongard, M. S. Smirnov and L. Friedrich (Moscow), working on extra-foveal colour-matching, $10^{\circ}$ out from the fovea, by the method of successive instead of juxtaposed presentation of stimuli, conclude that colour-vision there is essentially four-dimensional, not three-dimensional, the fourth fundamental spectral sensitivity being that of the rod mechanism. Matches made using four primaries were stable under variations of field intensity and adaptation while the approximate matches obtainable with three primaries were not.

The matching of colours produced by stimuli applied to different retinal areas, in the same or in opposite eyes, which are in different states of colour adaptation has bean actively studied in recent years, the case when only foveal (rod-free) areas are used being of particular importance. New measurements by E. N. Yustova (Moscow) of the colour changes as the foveal retina adapts to the stimulus colour itself showed the very large effects produced. In an attempt to explain a large class of these phenomens, D. L. MacAdam (Eastman Kodak Research Laboratory) was led in 1955 to postulate the operation of up to six independent spectral sensitivities in foveal vision. A new analysis by $R$. W. G. Hunt (Kodak Research Laboratory) showed that three may still suffice if signals in the optic nerve are not linearly related to the photochemical absorptions in the endorgans and if the non-linearity depends in a suitable way on the colour-adaptation of the retinal area. In his contribution to this symposium, MacAdam adopted a point of view close to Hunt's and with the aid of some specific hypotheses, which seem to involve the transmission of the arithmetic differences of the responses (non-linear) of different receptors, he was able to explain his original results on colouradaptation as well as some other psychophysical data.

Several contributors stressed the importance for colour perception of the time during which the retina is exposed to a particular pattern of stimulation (C. D. Farnsworth, U.S. Naval Medical Research Laboratory). The extreme situation is reached when, by suitable optical devices, the displacements of a retinal image produced by involuntary eye movements are nullified. R. W. Ditchburn (Reading), summarizing progress in this type of experiment, showed that a desaturation of hues-in some circumstances complete-may occur when stabilization of the retinal image is not sufficiently perfect to bring about complete loss of form vision.
Although only two papers dealt with questions of noise and quantum fluctuations in retinal response, the lively discussion provoked by the views and calculations of $\mathrm{H}$. Barlow (Cambridge) on the intrinsic noise in cone mechanisms showed the continuing interest in this subject.

Brief commentaries on the essentials of all colour theories (Y. LeGrand, Paris) and on subjective colour measurement (W. D. Wright, Imperial College) provided a suitable introduction to the more detailed contributions reviewed above. The proceedings-papers and discussions-will be published shortly by H.M. Stationery Office.

W. S. STILES

\section{SAFETY IN THE CHEMICAL INDUSTRY}

A SYMPOSIUM on "Safety in the Chemical Industry", arranged by the London Section of the Society of Chemical Industry, was held during October 14-15 at the Royal Institution. The chemical industry covers an enormously wide field of activity, and safety within it bears a direct relationship to manufacturing and handling, to safe transport and further handling by those who formulate and process the products of the primary producer.

In opening the symposium, Sir George Barnett, H.M. chief inspector of factories, dealt with the frequency rate of accidents in the chemical industry and mentioned that the figure for the chemical industry as a whole is $1 \cdot 43$ por 100,000 man-hours worked, as compared with 1.73 for the whole of industry. He also pointed out that a number of the larger chemical firms had frequency rates which were far better than the overall picture for the whole of the chemical industry.

$\mathrm{He}$ said that there were certain apparent dangers associated with the chemical industry, in the manufacture of poisonous substances, in the use of highpressure vessels, in dealing with now reagents and intermediate substances and the instability of a large number of chemical substances, and in the continual exploration of the field in which new reagents and new catalysts are being used. Sir George laid particular emphasis on the importance of chemical design in relation to the health of the process worker and the maintenance worker and also stressed the point that technical knowledge and ability, foresight and imagination are necessary in planning and design. Within recent years, much chemical plant had, in his view wisely, been housed in the open air, and he pointed out the advantages of remote control in the operation of many chemical plants.

The discipline of safety in the chemical industry is perhaps at least as far developed as in any other industry in Great Britain, and this discipline is reflected in the frequency rates of accidents. Safety, per se, may be divorced from the actual day-to-day training of engineers, chemists and physicists within the chemical industry, but mention was made of a special course which is now being started at the Imperial College of Science, where the general principles of safety will be taught.

With the rapid expansion of the chemical industry since the War, the problems of plant design, erection and start-up have become of major importance. The significance of dealing with materials of high toxicity was stressed by Mr. K. M. Curwen in his talk on the 